\title{
A modelling study of the impact of cirrus clouds on the moisture budget of the upper troposphere
}

\author{
S. Fueglistaler and M. B. Baker \\ University of Washington, Seattle, USA \\ Received: 7 September 2005 - Published in Atmos. Chem. Phys. Discuss.: 10 October 2005 \\ Revised: 21 February 2006 - Accepted: 1 March 2006 - Published: 4 May 2006
}

\begin{abstract}
We present a modelling study of the effect of cirrus clouds on the moisture budget of the layer wherein the cloud formed. Our framework simplifies many aspects of cloud microphysics and collapses the problem of sedimentation onto a 0 -dimensional box model, but retains essential feedbacks between saturation mixing ratio, particle growth, and water removal through particle sedimentation. The water budget is described by two coupled first-order differential equations for dimensionless particle number density and saturation point temperature, where the parameters defining the system (layer depth, reference temperature, amplitude and time scale of temperature perturbation and inital particle number density, which may or may not be a function of reference temperature and cooling rate) are encapsulated in a single coefficient. This allows us to scale the results to a broad range of atmospheric conditions, and to test sensitivities. Results of the moisture budget calculations are presented for a range of atmospheric conditions ( $T: 238-205 \mathrm{~K}$; $p$ : $325-180 \mathrm{hPa}$ ) and a range of time scales $\tau_{\mathrm{T}}$ of the temperature perturbation that induces the cloud formation. The cirrus clouds are found to efficiently remove water for $\tau_{\mathrm{T}}$ longer than a few hours, with longer perturbations $\left(\tau_{\mathrm{T}} \gtrsim 10 \mathrm{~h}\right)$ required at lower temperatures $(T \lesssim 210 \mathrm{~K})$. Conversely, we find that temperature perturbations of duration order $1 \mathrm{~h}$ and less (a typical timescale for e.g., gravity waves) do not efficiently dehydrate over most of the upper troposphere. A consequence is that (for particle densities typical of current cirrus clouds) the assumption of complete dehydration to the saturation mixing ratio may yield valid predictions for upper tropospheric moisture distributions if it is based on the large scale temperature field, but this assumption is not necessarily valid if it is based on smaller scale temperature fields.
\end{abstract}

Correspondence to: S. Fueglistaler

(stefan@atmos.washington.edu)

\section{Introduction}

Water vapor is the atmosphere's most important greenhouse gas (e.g. Held and Soden, 2000) and condensed water in clouds strongly affects the Earth's radiation balance. Hence, understanding water distribution in, and transport through, the atmosphere is an important aspect of understanding the climate system. Here, we present a modelling study that addresses the impacts of cirrus clouds on the moisture budget of upper tropospheric air masses. Specifically, we explore the relation between the time scales of atmospheric motions that induce clouds and the time scales of water removal by the sedimenting ice particles, and the efficiency of cirrus cloud dehydration at various levels of the upper troposphere.

The formation of ice particles through homogeneous or heterogeneous nucleation, their subsequent growth and sedimentation provide a challenge to any modelling effort, and the net effect of a cirrus cloud on the water vapor budget of an atmospheric layer often strongly depends on a large number of parameters and boundary conditions. These include, but are not restricted to: temperature and relative humidity profiles, aerosol concentrations and the air masses' temperature history, on both long timescales (determining the available amount of water) and short (relevant for nucleation). Consequently, a broad range of phenomena are observed in the atmosphere, often associated with very specific atmospheric conditions. For example, Hall and Pruppacher (1976) showed how ice particles could survive a fall over several kilometers in subsaturated air, giving rise to so-called "fall-streaks". Their study, however, also showed that such phenomena can occur for a limited range of relative humidity profiles and initial particle sizes only.

Given the strong and non-linear coupling of particle nucleation, particle number density, growth/evaporation of particles, and sedimentation fall speeds, models may be required to track individual ice crystals as they fall through the atmosphere in order to accurately model cloud evolution

Published by Copernicus GmbH on behalf of the European Geosciences Union. 
and the vertical redistribution of water. Such models have been successfully applied to denitrification in the polar vortex (Fueglistaler et al., 2002) and for the modelling of thin cirrus near the tropical tropopause (Luo et al., 2003; Jensen and Pfister, 2004). However, since these models were tailored to address very specific questions, they are not suited to address more general questions regarding typical dehydration timescales of cirrus clouds. Studies addressing issues of moisture transport on the large scale therefore often employ highly simplified cloud physics (e.g. Pierrehumbert, 1998; Dessler and Sherwood, 2000; Fueglistaler et al., 2005), which introduces uncertainty in their results regarding the order of magnitude of errors brought in by these simplifications.

In order to obtain a better understanding of typical timescales of cirrus clouds, and their implications for modelling moisture transport, we employ a simplified model of the water budget of an upper tropospheric layer. We neglect details of the microphysics but retain the important couplings between particle growth/evaporation, saturation mixing ratio and water depletion through sedimentation of particles. We consider only cases where ice crystals nucleate in the layer of interest, and exclude cases (for example convective anvils) where nucleation and growth occurr under very different atmospheric conditions than particle sedimentation.

The model calculations are used to evaluate a) whether the dehydration is "fast" or "slow"), and b) the "efficiency" of the cloud to dehydrate the air mass, where "efficient" means that the layers' terminal mixing ratio is close to, or equal to, the minimum saturation mixing ratio of its temperature history.

Section 2 describes the model physics. Section 3 shows the evolution of the layer water budget for specific scenarios. In Sect. 4 we define a dehydration efficiency and calculate it for the range of reference temperatures and perturbation time scales typical of the upper troposphere. Section 5 provides a discussion of the applicability of the model results to the atmosphere, and of the model limitations. Finally, Sect. 6 summarizes the conclusions.

\section{Model description and model parameters}

The 0 -dimensional model employed here describes the water budget of an atmospheric layer with depth $h$, wherein particles nucleate. Note that "h" is not the "cloud depth", i.e. the total layer wherein ice particles exist. Due to sedimentation of ice particles, and depending on relative humidity profiles and particle sizes, the "cloud depth" can be much larger than the layer wherein the particles formed. Most of the results shown are based on the assumption of homogeneous ice nucleation following the parameterization based on water activity by Koop et al. (2000), where we used simplifications similar to the "fast growth" scenario by Kärcher and Lohmann (2002). However, in Sect. 4.3 we show that the overall char- acter of these results is not very sensitive to this assumption, and that our conclusions can be scaled to include heterogeneous nucleation.

Once formed, particles are assumed to be in equilibrium with the surrounding gas phase, an assumption well justified for the cases discussed here with relatively high particle number densities, such that diffusive equilibration is a fast process (time scale of minutes) compared to the time scale of the temperature perturbations (hours). Recent observations suggest some supersaturation even in the presence of considerable ice surface area density (Jensen et al., 2005), however, there is no consensus yet as to what may be the responsible mechanism. We therefore consider it at this point premature to include such an effect in our simplified model. The available water vapor is equally distributed among all particles in the layer, giving rise to a uniform, monodisperse particle size distribution in the layer. Hence, the model does not resolve the complexities of a condensed water flux resulting from ice particles with a spectrum of fall velocities, but it does retain the important coupling between temperature (and hence saturation mixing ratio) and particle size and associated fall speed.

The water loss due to gravitational settling of the particles is calculated using the Locatelli and Hobbs (1974) parameterization of fall velocities as function of crystal size. Once a cloud is present in the layer, no new ice nucleation is assumed to occur, and the particle number density decreases as the particles fall out of the layer. This may lead to a small bias in particle sizes towards larger values (since the available water vapor during the cooling phase is distributed among fewer particles), and consequently the condensed water flux is somewhat overestimated.

As the temperature perturbation of the air mass is prescribed, the calculations ignore radiative impacts on the clouds. Possible impacts of radiation on the conclusions derived from the model calculations are discussed in Sect. 5.1.2.

The model formalism is presented in the Appendix, and we discuss here some input parameters that deserve special attention.

\subsection{Temperature perturbation $\delta T$}

We prescribe isobaric temperature perturbations (the much weaker linear dependence on pressure of the saturation mixing ratio is neglected for simplicity) leading to the formation of cirrus clouds as harmonic oscillations around a reference temperature $T_{\text {ref }}$. The period of the oscillation (and hence the time scale of the temperature perturbation) is $\tau_{\mathrm{T}}, \Delta T$ is its amplitude, and $\delta T(t)$ denotes the temperature deviation at time $t$ from the reference temperature $T_{\text {ref }}$, i.e.

$$
\begin{aligned}
\delta T(t) & =T(t)-T_{\text {ref }} \\
& =-\Delta T \cdot \sin \left(2 \pi t / \tau_{T}\right), 0 \leq t \leq \tau_{\mathrm{T}} / 2 .
\end{aligned}
$$


Note that we have chosen to restrict $t$ to the "cold phase" of the temperature oscillation only. For these computations we associate a pressure $p\left(T_{\text {ref }}\right)$ with each value of $T_{\text {ref }}$ taken from a typical measured sounding during cirrus events at the Atmospheric Radiation Measurement Program (ARM) site Oklahoma.

The dependence of the model equations on $\Delta T$ is complex because this parameters enters the model in various ways. In most calculations we use $\Delta T=2 \mathrm{~K}$, a simplification in order to keep the number of free variables as low as possible. A discussion of results for varying $\Delta T$ is provided in Sect. 4.1.

\subsection{Layer depth $h$}

Obviously, there is not a single number that can be assigned to the layer depth $h$ based on firm physical grounds. Note that $h$ is not the thickness of the cloud; rather it is merely the scale of the layer in which we assume particles nucleate, grow and eventually leave through sedimentation. As stated before, the (visible) "cloud depth" comprises this layer plus the layer below it, which particles fall into, but in which they do not immediately evaporate because of conditions close to saturation. The treatment of the layer as a homogeneous "box" requires sufficiently homogeneous conditions within that box, which puts an upper bound to $h$ of a few hundred meters. A deeper layer would not only have very inhomogeneous temperatures due to the vertical lapse rate or inhomogeneities in relative humidity, but would also be strongly affected by vertical wind shear over the time scales considered here.

We have therefore assigned a value of $h=300 \mathrm{~m}$, which we consider as a sensible value, with a possible range at different atmospheric conditions of about a factor two. Section 4.3 shows how the results may be scaled to account for variability in $h$.

\subsection{Initial particle number density $n_{0}$}

Homogeneous nucleation of ice particles depends on the cooling rate at the time when the critical saturation is reached, and therefore inherently depends on short time scale temperature fluctuations. In order to simplify and render the calculations comparable with one another, we specify conditions at time $t=0$ (when $\delta T=0$ ) such that nucleation immediately sets in. Hence, the cooling rates at the time of nucleation are

$\mathrm{d} T / \mathrm{d} t(t=0)=-\Delta T \frac{2 \pi}{\tau}[\mathrm{K} / \mathrm{h}]$,

that is for an amplitude $\Delta T=2 \mathrm{~K}$ and a period $\tau_{\mathrm{T}}=2 \mathrm{~h}$ the cooling rate at the time of nucleation is $\sim-6 \mathrm{~K} / \mathrm{h}$.

We compute the initial ice particle number density $n_{0}\left[\mathrm{~cm}^{-3}\right]$ at each temperature for these cooling rates from the Kärcher and Lohmann (2002) parameterization. This parameterization yields a maximum of $n_{0} \approx 1.0 \mathrm{~cm}^{-3}$ at $T_{\text {ref }}=205 \mathrm{~K}, p=179 \mathrm{hPa}$ and $\tau_{\mathrm{T}}=1 \mathrm{~h}$, and a minimum of $n_{0} \approx 0.001 \mathrm{~cm}^{-3}$ at $T_{\text {ref }}=238 \mathrm{~K}, p=325 \mathrm{hPa}$ and $\tau_{\mathrm{T}}=10 \mathrm{~h}$.
These values compare well with observations, with a typical value of $10^{-1} \mathrm{~cm}^{-3}$, ranging from $10^{-3} \mathrm{~cm}^{-3}$ to $10^{1} \mathrm{~cm}^{-3}$ (see e.g. observations compiled by Dowling and Radke, 1990).

Note that coupling of particle nucleation to the cooling rate derived from the temperature perturbation leads to perhaps artificially small values of $n_{0}$ for very long time scales (i.e. $\tau_{\mathrm{T}} \sim 10 \mathrm{~h}$ ). In reality, shorter period temperature perturbations with higher cooling rates (see e.g. Hoyle et al., 2005) and heterogeneous nucleation would then dominate the particle formation. Consequently, the calculations tend to underestimate $n_{0}$, and hence overestimate the sedimentation velocity, for long $\tau_{\mathrm{T}}$. This point will be discussed in Sect. 4.3 below.

\subsection{The scaling parameter $P$}

As shown in the Appendix, in our approximation the differential equations governing the system at fixed $\Delta T$ depend on a single nondimensional parameter $P$ that encapsulates the temperature perturbation time scale $\tau_{\mathrm{T}}$, layer depth $h$, and initial particle number density $n_{0}$ :

$$
P \equiv \frac{\tau_{\mathrm{T}}}{2 \pi h / v\left(r_{0}\right)} \equiv \frac{\tau_{\mathrm{T}}}{2 \pi \tau_{\text {fall }}},
$$

where $v(r)$ is the sedimentation velocity of a particle of effective size $r$, and $r_{0}$ is a particle radius scale involving the initial ice particle density and $\Delta T$ (see Appendix). Since in our simple model $P$ governs the results, a wide range of values of the input parameters can be explored by simply relating them to the associated value of $P$ and looking-up the results shown below for that specific value of $P$.

Figure 1 shows the dependence of $P$ for $\Delta T=2 \mathrm{~K}$ on $\tau_{\mathrm{T}}$ and reference temperature $T_{\text {ref }}$ for values characteristic of the upper troposphere. The two time scales are similar ( $\tau_{\mathrm{T}} \approx \tau_{\text {fall }}$ ) for $P \approx 0.16$. For $P$ much greater than this value the sedimentation time scale is much shorter than $\tau_{\mathrm{T}}$, and we say that the dehydration is "fast". Conversely, for $P \lesssim 0.1$ the sedimentation time scale is long compared to $\tau_{\mathrm{T}}$, and the dehydration is "slow". Figure 1 shows that the conditions in the upper troposphere encompass both regimes, with values of $P$ ranging from order 0.01 to 10 . Under upper tropospheric conditions cirrus dehydration is in the fast regime $(P>>0.16)$ for all $\tau_{\mathrm{T}} \gtrsim 3 \mathrm{~h}$ at $T_{\text {ref }} \approx 235 \mathrm{~K}$, increasing to all $\tau_{\mathrm{T}} \gtrsim 10 \mathrm{~h}$ at $T_{\text {ref }} \approx 210 \mathrm{~K}$. Similarly, cirrus dehydration is in the slow regime for all $\tau_{\mathrm{T}} \lesssim 1 \mathrm{~h}$ at $T_{\text {ref }} \approx 235 \mathrm{~K}$, increasing to all $\tau_{\mathrm{T}} \lesssim 4 \mathrm{~h}$ at $T_{\text {ref }} \approx 210 \mathrm{~K}$.

Figure 2 shows the contours of $P$ for fixed $n_{0}=0.1 \mathrm{~cm}^{3}$. The overall character of the $P$ contour pattern is very similar to that of the homogeneous nucleation scenario as shown in Fig. 1. For high $T_{\text {ref }}$ and/or long $\tau_{\mathrm{T}}$ the number of ice crystals nucleated homogeneously is less than $0.1 \mathrm{~cm}^{-3}$, so $P$ contours have moved to the right in Fig. 2. At lower $T_{\text {ref }}$ and/or shorter $\tau_{\mathrm{T}}$ the reverse is true; $P$ contours have moved to the left. 


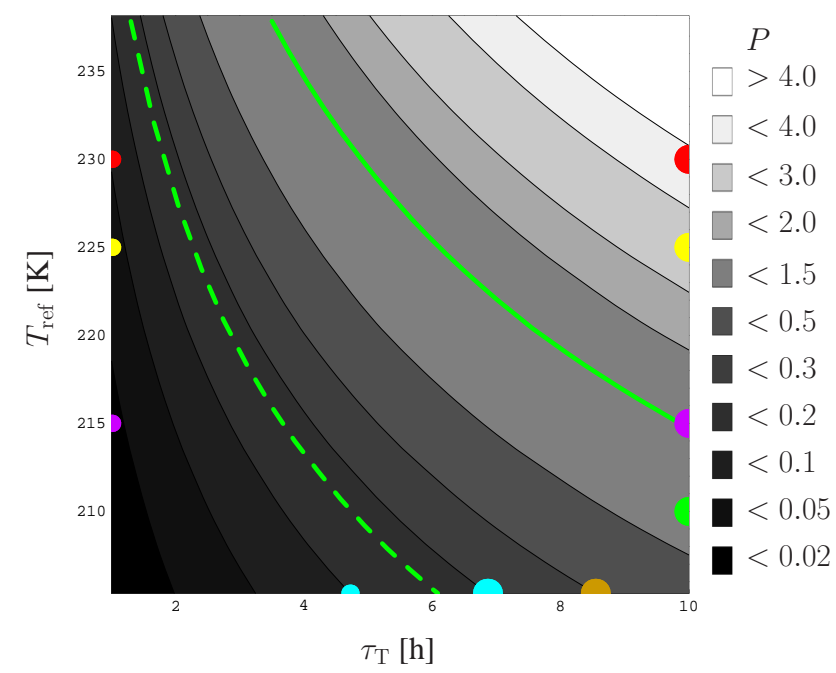

Fig. 1. The non-dimensional parameter $P$ as function of $T_{\text {ref }}$ and $\tau_{\mathrm{T}}$ at $\Delta T=2 K$. Higher $P$ values correspond to lighter gray values. The green dashed curve is $P=0.16$ and the green solid curve is $P=1$. Colored dots are the $T_{\text {ref }}, \tau_{T}$ values for cases discussed in Sect. 3. The $P$ values associated with these dots are as follows: starting from the small red dot on the upper left and moving counterclockwise; $P=.05, .035, .015,0.1,0.2,0.3,0.64,1.0,2.5,3.8$. Small dots correspond to $P \leq 0.16$ (slow regime) and large dots to larger $P$ values (fast regime).

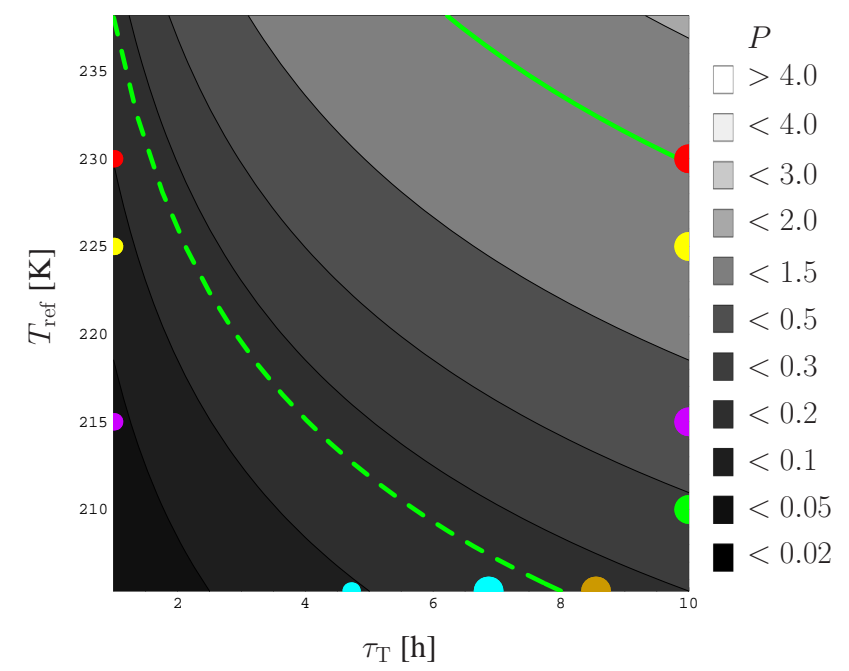

Fig. 2. Contours of $P$ at fixed $\Delta T=2 K$ for fixed $n_{0}=0.1 \mathrm{~cm}^{-3}$. Color coding and contours as in Fig. 1.

The impact of increasing $n_{0}$ on $P$ is seen in Fig. 3, where the $P=0.16$ contour is shown for fixed $n_{0}=0.1,0.5$ and $1 \mathrm{~cm}^{-3}$. For the lowest $n_{0}$ value only the lower left hand portion of the $\tau_{\mathrm{T}}, T_{\text {ref }}$ graph falls into the slow regime, whereas for only a factor of 10 increase in particle density most of the

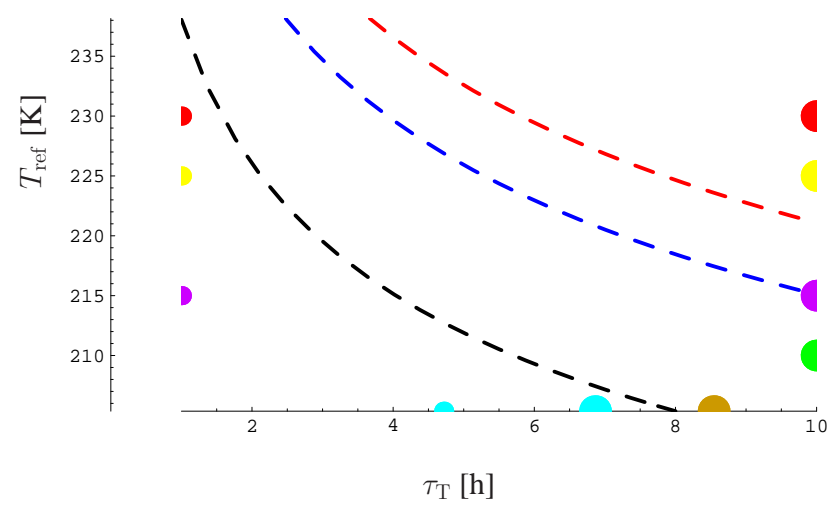

Fig. 3. $P=0.16$ contour for fixed $n_{0}=0.1 \mathrm{~cm}^{-3}$ (black), $n_{0}=0.5 \mathrm{~cm}^{-3}$ (blue) and $n_{0}=1 \mathrm{~cm}^{-3}$, (red). As $n_{0}$ increases, more and more of the $\left(T_{\text {ref }}, \tau_{\mathrm{T}}\right)$ range falls into the $P \leq 0.16$ (slow) regime, where clouds persist for most (or all) of the perturbation period.

upper troposphere would correspond to values in this regime. This implies an important role for ice nuclei in the hydrological cycle of the upper troposphere.

\section{Cloud evolution}

To facilitate comparison among model results at different reference temperatures, we express the total water content of the cloud layer in terms of the saturation point temperature $T^{*}$ and its difference from the reference temperature, $\delta T^{*}(t) \equiv T^{*}(t)-T_{\text {ref }}$, rather than in terms of the mixing ratio in the layer.

At a given pressure and given total water content the saturation point temperature $T^{*}$ is the temperature at which there would be no condensate and the air would be exactly saturated with respect to ice (Betts et al., 1982) (the "frost point temperature").

Results are presented in terms of the non-dimensionalized deviations of temperature and of saturation point temperature from $T_{\text {ref }}$,

$\delta \hat{T}(t) \equiv \delta T(t) / \Delta T$

and

$\delta \hat{T}^{*}(t) \equiv \delta T^{*}(t) / \Delta T$.

The number density of ice particles left in the layer at time $t$ is $n(t)$ and the fraction of originally nucleated particles that remains in the layer is $\hat{n}(t) \equiv n(t) / n_{0}$. (see also Appendix).

Figure 4 shows the evolution of $\delta \hat{T}$ and $\delta \hat{T}^{*}$ as functions of the non-dimensionalized time $\hat{t} \equiv 2 \pi t / \tau_{\mathrm{T}}$ for each of the $\left(T_{\text {ref }}, \tau_{\mathrm{T}}\right)$ conditions shown by a colored dot in Figs. 1 and 2. 


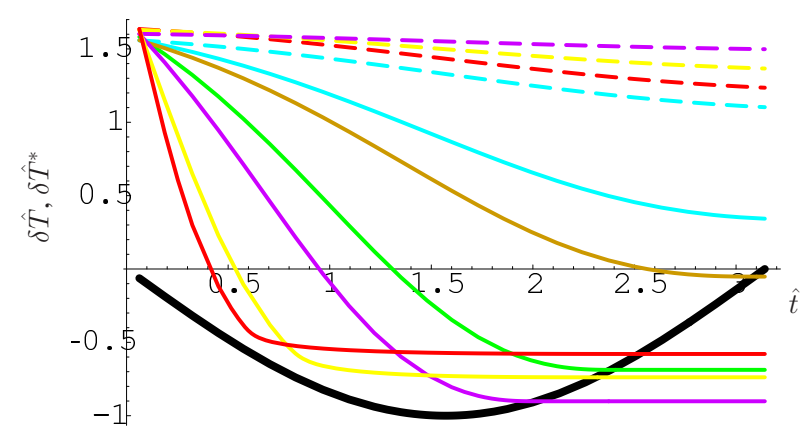

Fig. 4. Evolution of nondimensionalized temperature $\delta \hat{T}(\hat{t})$ (bold black) and of nondimensionalized saturation point temperature $\delta \hat{T}^{*}(\hat{t})$ over a single cooling event. Each curve corresponds to a $\left(T_{\text {ref }}, \tau_{\mathrm{T}}\right)$ pair shown by a dot of the same color in Fig. 1 . Dashed curves correspond to the small dots in that figure and solid curves to the larger ones.

All cases for which $P<0.16$ (dashed curves) are in the slow regime. That is, particle sedimentation is slow compared to the time scale of the temperature perturbation. Figure 4 shows that for these cases the layer, indeed, slowly dehydrates, and that the total moisture at $t=\tau_{\mathrm{T}} / 2$ (before the onset of the warming phase) is only marginally reduced by the cirrus cloud. Figure 4 further shows how the character of the cloud impact on layer dehydration changes in the transition region $P \approx 0.16$ where the two time scales $\tau_{\mathrm{T}}$ and $\tau_{\text {fall }}$ are similar. (See the two turquoise curves, corresponding to $P=0.1$ and $P=0.2$ in these figures.) The cases with $P \geq 1$ are clearly in the fast regime.

All cases with $P \geq 1$ experience complete fall-out before $t=\tau_{\mathrm{T}} / 2$; however, their terminal moisture content shows an interesting, non-monotonic dependence on $P$. One might expect that for faster dehydration the final moisture content should always decrease. Figure 4 shows that for the cases with $P \geq 1$ (purple, yellow and red; solid curves) this is not the case. Rather, in these cases the fall-out is so fast that all particles have left the layer before the temperature minimum is reached. This is in part due to the low bias of the calculated $n_{0}$ at long time scales $\tau_{\mathrm{T}}$ as discussed in Sect. 2.3, and Sect. 4.3 shows that this behaviour largely vanishes when the bias in $n_{0}$ is corrected.

Our discussion of specific scenarios has highlighted the role of $P$ in determining whether dehydration is fast or slow. We have touched upon the relation between $P$ and the final moisture content at time $t=\tau_{\mathrm{T}} / 2$, which is the focus of the next section.

\section{Dehydration efficiency}

We define the dehydration efficiency $\epsilon$ of a cloud as the amount of water actually removed by the cloud during a given isobaric cooling perturbation, divided by the maximum

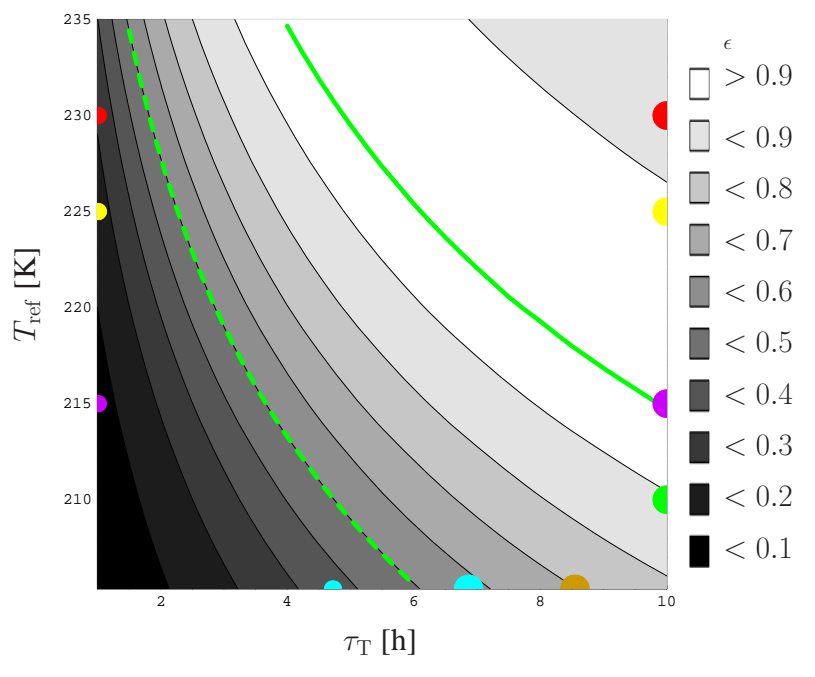

Fig. 5. The dehydration efficiency $\epsilon$ (see Eq. 6) for $\Delta T=2 \mathrm{~K}$ for the range of $T_{\text {ref }}$ and $\tau_{\mathrm{T}}$ shown in Fig. $1 . P=0.16,1$ (green curves) and colored dots as in Fig. 1.

water made available for gravitational removal by that perturbation. Using the previously introduced notation, $\epsilon$ is defined as

$\epsilon \equiv \frac{Q\left(T^{*}(\hat{t}=\pi)\right)-Q\left(T^{*}(0)\right)}{Q\left(T_{\mathrm{ref}}-\Delta T\right)-Q\left(T^{*}(0)\right)}$

where $Q$ is the total water vapor mixing ratio.

In cases for which $\epsilon \approx 1$, the cloud event removes the maximum possible water from the layer; i.e., at the end of the event the mixing ratio is close to the saturation mixing ratio at $T=T_{\text {ref }}-\Delta T$, whereas $\epsilon \approx 0$ indicates that the most of the ice particles re-evaporate within the layer.

Figure 5 shows that the cirrus clouds efficiently dehydrate the layer for high temperatures and time-scales $\tau_{\mathrm{T}}$ longer than a few hours. As $\tau_{\mathrm{T}}$ gets shorter, the dehydration efficiency decreases rapidly (depending on $T_{\text {ref }}$ ), and temperature perturbations shorter than $\sim 1 \mathrm{~h}$ are too short to allow the particles to fall significantly at all. For fixed $\Delta T \epsilon$ essentially depends on the governing parameter $P$ only (see Appendix). Figure 5 shows this in that the contours of $\epsilon$ parallel those of $P$. The figure shows the previously noted increase of $\epsilon$ as $P$ gets larger, with a slight decrease of $\epsilon$ at very high values of $P$.

Note that the $P=0.16$ curve lies very close to the $\epsilon=0.5$ contour, so that the fast and slow regimes correspond to $\epsilon>0.5$ and $\epsilon<0.5$, respectively. This coincidence is closest for $\Delta T=2 \mathrm{~K}$ but does not change greatly for other values of $\Delta T$. 


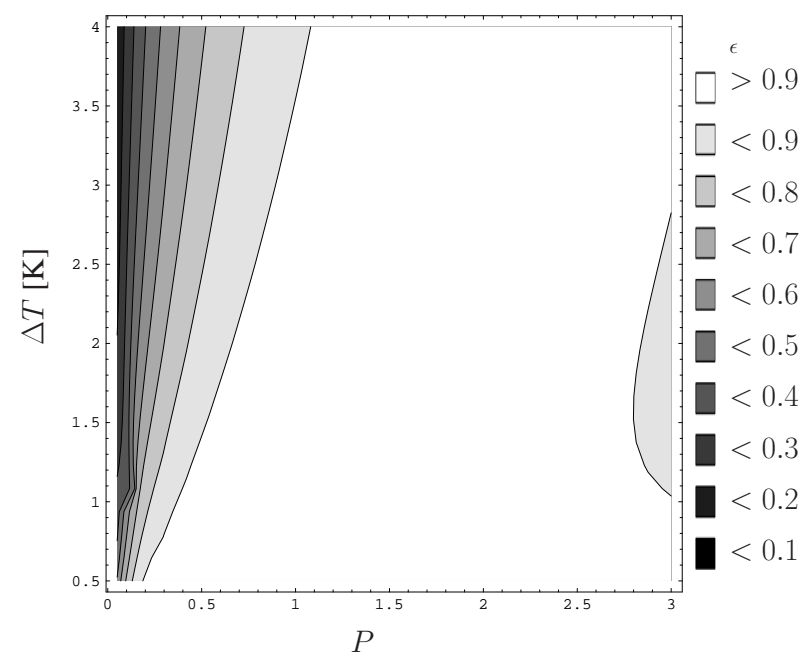

Fig. 6. Dehydration efficiency $\epsilon$ for a range of values of $\Delta T$ and $P$, contours are as in Fig. 5.

4.1 Dependence of the dehydration efficiency on $T_{\text {ref }}$ and $\Delta T$

From the definition of $\epsilon$ it can be seen that it is almost independent of $T_{\text {ref }}$ at constant $P$. For $\delta T^{*}, \delta T \ll T_{\text {ref }}$, we can linearize the expression for $\epsilon$ in Eq. (6), to yield

$\epsilon \approx \frac{\delta \hat{T}^{*}(\hat{t}=0)-\delta \hat{T}^{*}(\hat{t}=\pi)}{1+\delta \hat{T}^{*}(\hat{t}=0)}$

In this equation $T_{\text {ref }}$ enters implicitly through its influence on the value of $\delta \hat{T}^{*}$ at cloud initiation $(\hat{t}=0)$. The term $\delta \hat{T}^{*}(\hat{t}=0)$ represents the supersaturation needed for nucleation to occur.

The dehydration efficiency depends on $\Delta T$ in the following ways. The sedimentation velocity scale $v\left(r_{0}\right)$, and hence $P$ (Eq. 3), depend on the maximum available condensedphase water, as well as on the initial particle number density $n_{0}$. The maximum available condensate depends on $T_{\text {ref }}$ and $\Delta T$. Further, $n_{0}$ is a function of the cooling rate (and hence $\Delta T$, see Eq. 2). Finally, the scaled initial condition, $\delta \hat{T}^{*}(\hat{t}=0)$, also depends on $\Delta T$. Thus both the numerator and denominator of Eq. (7) depend on $\Delta T$.

Figure 6 shows the dehydration efficiency as function of $P$ and $\Delta T$. For $P>1$ the efficiency is near unity for all $\Delta T$, but for small $P$ the efficiency decreases with increasing $\Delta T$; the amount of water that falls out of the cloud does not increase as fast as does the maximum potential water loss as $\Delta T$ increases.

For $\Delta T=2 \mathrm{~K}, \epsilon(P)$ is well fit by the polynomial

$\epsilon(P)=3.58 P-6.99 P^{2}+8.57 P^{3}-6.22 P^{4}+2.38 P^{5}-0.36 P^{6} .(8)$

This fit may be useful for studies of upper tropospheric humidity involving dehydration due to cirrus clouds. The

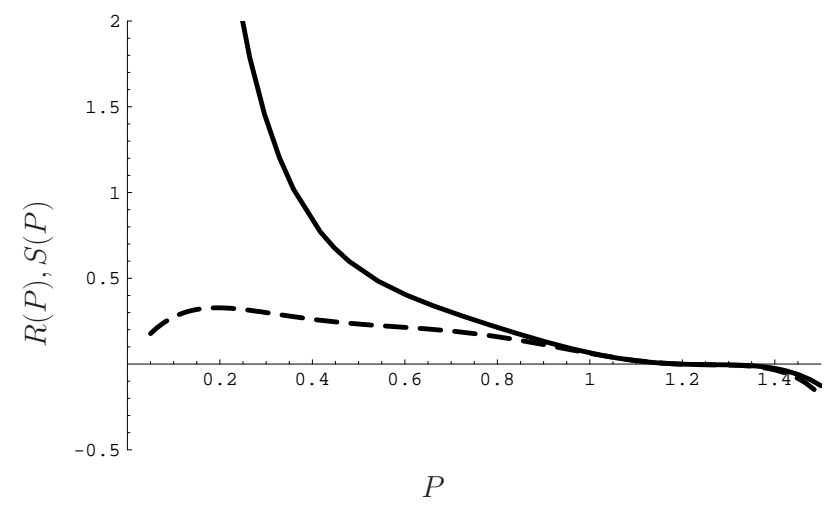

Fig. 7. Two measures (see text) of the variation in dehydration efficiency $\epsilon$ to variations in $P$ at constant $\Delta T=2 \mathrm{~K}$ : the response $R(p) \equiv d \epsilon / d \ln P($ dashed $), \quad$ and $\quad$ sensitivity $\quad S(p) \equiv d(\ln \epsilon) / d P$ (solid).

change in total water content of an atmospheric layer due to clouds within it can be estimated as follows: When the critical supersaturation for nucleation is reached (no matter what its value is assumed to be), determine $T_{\text {ref }}$ and $\tau_{\mathrm{T}}$ (approximated for example by twice the time span until temperatures exceed $T_{\text {ref }}$ again). Next, $\tau_{\text {fall }}$ is specified based on assumed $n_{0}$ and $h$ (see Appendix). Now $P$ is given by Eq. (3) which is inserted into the fit for $\epsilon$ (Eq. 8). Finally, Eq. (6) is rearranged to obtain the removed water

$$
\begin{aligned}
\Delta Q & =Q\left(T^{*}(\hat{t}=\pi)\right)-Q\left(T^{*}(0)\right) \\
& =\epsilon \cdot\left(Q\left(T_{\mathrm{ref}}-\Delta T\right)-Q\left(T^{*}(0)\right)\right) .
\end{aligned}
$$

\subsection{Sensitivity of $\epsilon$ to changes in $P$}

For two reasons we are interested in examining the variations in $\epsilon$ due to uncertainties in $P$. First, the values of the parameters that determine $P$ are not precisely known, and hence introduce an uncertainty. Second, processes in the climate system that would systematically change the parameters that determine $P$, for example cloud particle number densities, would induce a change in $\epsilon$. These variations of $\epsilon$ to changes in $P$ can easily be derived from Eq. (8).

Figure 7 (solid line) shows the classically defined sensitivity $\mathrm{d} l n \epsilon / \mathrm{dP}$, i.e. the fractional change of $\epsilon$ due to a given change in $P$. Evidently, the sensitivity is largest at small $P$, indicating that the dehydration efficiency of clouds in the slow regime is most sensitive, whereas the dehydration efficiciency in the fast regime is largely insensitive to changes in $P$.

A somewhat different, but equally useful, quantity is the response $d \epsilon / d \ln P$ (not to be confused with the previous "sensitivity") of $\epsilon$ to a relative change in $P$ (Fig. 7, dashed line). One can argue that it is not so much the relative change in $\epsilon$, but rather its absolute change, that is relevant here (because, for example, the climate system reacts to absolute 
changes in $\epsilon$ ). Further, for at least some of the parameters determining $P$, the values may be known to within a certain factor, rather than an absolute value. The "response" defined in this way shows a maximum at $P \approx 0.16$, i.e. where the two timescales $\tau_{\mathrm{T}}$ and $\tau_{\text {fall }}$ are equal. The response decreases as $P$ approaches both the slow and fast regime. In other words, dehydration remains inefficient in the slow regime, and remains efficient in the fast regime.

\subsection{Scaling arguments}

The results presented in the previous section were evaluated for $\Delta T=2 \mathrm{~K}, h=300 \mathrm{~m}$ and particle number densities for homogeneous nucleation parameterized in terms of maximum cooling rates. We now show that these results can be scaled to provide results for other parameter combinations.

In our simple model the parameters $P$ and, to a lesser extent, $\Delta T$, govern the evolution of the scaled saturation temperature $\left(\hat{T}^{*}\right)$. Hence, for a given $\Delta T$ any parameter combination that yields the same value for $P$ will have the same solution.

For example, the dehydration efficiency for an assumed depth $h=600 \mathrm{~m}$ instead of $300 \mathrm{~m}$ is readily read off from Fig. 6 by scaling $\tau_{\mathrm{T}}$ correspondingly with a factor 2 . The dehydration efficiency at a given reference temperature and a specific $\tau_{X}$ (for example $5 \mathrm{~h}$ ) for the deeper cloud is then read off from Fig. 6 at the same reference temperature, but at $\tau_{\mathrm{T}}=2 \times \tau_{X}$ (in this case $10 \mathrm{~h}$ ).

We have mentioned above that assuming homogeneous nucleation as function of $\delta T(t)$ yields very low values of $n_{0}$ at relatively high temperatures and/or long $\tau_{\mathrm{T}}$, which is a bit nonphysical, since it ignores the role of the (superimposed) high frequency temperature perturbations (Hoyle et al., 2005) and neglects any contribution of ice nuclei. These low $n_{0}$ values then artificially inflate the value of $P$ leading to an apparent decrease in $\epsilon$ at long $\tau_{\mathrm{T}}$. Using the scaling properties of $P$, we may determine the impact of higher $n_{0}$ for these cases.

To determine the dehydration efficiency at $n_{0}=0.1 \mathrm{~cm}^{-3}$ for given $T_{\text {ref }}, \tau_{\mathrm{T}}$ ), we read off the $P$-value for those parameter values from Fig. 2 , which we can denote $P=P_{0}$. Now, using Fig. 1 we find that $\tau_{\mathrm{T}}$ at the same reference temperature for which $P=P_{0}$. Thus we have two equivalent situations, in which the difference in $n_{0}$ is compensated by a difference in $\tau_{\mathrm{T}}$. For example, to find the dehydration efficiency for the conditions corresponding to the large red dot of Fig. $2\left(T_{\text {ref }}=230 \mathrm{~K}, \tau_{\mathrm{T}}=10 \mathrm{~h}, P_{0}=1\right)$, we find the equivalent $\left(P_{0}=1\right)$ homogeneous nucleation case in Fig. 1. This obtains for $T_{\text {ref }}=230 \mathrm{~K}, \tau_{\mathrm{T}} \approx 5 \mathrm{~h}$, for which the dehydration efficiency (Fig. 6) $\epsilon \approx 1$, i.e. higher than in the homogenous nucleation (coupled to the cooling rate proportional to $\tau_{\mathrm{T}}$ ) scenario.

Thus, a shift towards higher particle number densities induces a shift in $P$ towards lower values, mitigating the previously noted peculiarity of slightly decreasing efficiencies at long $\tau_{\mathrm{T}}$. The decrease in $\epsilon$ with $\tau_{\mathrm{T}}$ is thus shifted to very long temperature perturbations $\tau_{\mathrm{T}}>>10 \mathrm{~h}$, which is (for most cases) of little relevance since cloud formation and evolution would occur by superimposed shorter temperature perturbations. Note that for the cases where the homogeneous nucleation scenario yielded $n_{0}>0.1 \mathrm{~cm}^{-3}$, the shift is in the other direction, towards higher values of $P$ (faster). For example, the case $\tau_{\mathrm{T}}=1 \mathrm{~h}$ and $T_{\text {ref }}=230 \mathrm{~K}$ (small red dot) has $P \approx 0.05$ in the homogeneous nucleation scenario, and $P \gtrsim 0.1$ for $n_{0}=0.1 \mathrm{~cm}^{-3}$.

\section{Discussion}

The results of this modelling study do not allow us to directly deduce conclusions about atmospheric moisture distribution, or, for example, frequency of cirrus cloud occurrence. Rather, these quantities must be determined by specific studies that prescribe the dynamic regime (e.g. midlatitude frontogenesis, or subtropic large-scale descent) to determine the temperature history, and prescribe the water source terms (e.g. from moist convective updrafts, or mixing with moist layers). As pointed out in the introduction, the vertical cloud structure (and hence for example also the cloud's optical depth) might depend crucially on the relative humidities of the air masses below the layer studied here. If this layer were near saturation, one would expect a vertically thick cloud, whereas for a very dry layer the particles would immediately evaporate. Further, aspects such as cloud occurrence frequency may depend on the saturation mixing ratio required for nucleation and highly resolved temperature fields (e.g. Jensen and Pfister, 2004). In other words, cirrus cloud distributions and (vertical) structure depend crucially on the air masses' history in terms of temperature and moisture flux, and may be very sensitive to parameters such as particle number density or nucleation threshold.

What our scaling results, however, robustly predict, is that in the upper troposphere the atmosphere cannot hold more water than given by the saturation mixing ratio for temperature perturbations of order $1 \mathrm{~h}$ and longer at high temperatures $(230 \mathrm{~K})$, and longer than a few hours at very low temperatures $(205 \mathrm{~K})$. Conversely, typical sedimentation in cirrus clouds is too slow to substantially dehydrate upper tropospheric air masses for temperature perturbations shorter than these time scales. In practice, this means that the temperature field as resolved by large-scale atmospheric models combined with dehydration to the minimum saturation mixing ratio is a reasonable basis to study upper tropospheric moisture transport and distribution.

This has been implicitly assumed in previous studies which have shown empirically that model runs with complex and simple cloud microphysics yield similar results for the atmospheric moisture distribution. We believe that our study provides useful scaling arguments as to why this is the case in the upper troposphere. 


\subsection{Limitations}

\subsubsection{Particle size distribution}

The model assumes a monodisperse particle size distribution, a necessary simplification in order to keep the model simple and physically consistent. This leads to an underestimation of the dehydration arising from the larger particles, and an overestimation of that arising from smaller particles. In other words, the initial water flux (arising from the larger particles) is underestimated, and the fraction of condensed mass that re-evaporates within the layer (arising from the smaller particles) may be underestimated (leading to an overestimation of the dehydration efficiency $\epsilon$ ). These biases are obviously largest for cases with very broad size distributions, which, e.g. could arise due to the presence of a few heterogeneous ice nuclei that nucleate prior to the onset of homogeneous nucleation. For such cases a more detailed assessment using single particle models (e.g. Fueglistaler et al., 2002) is warranted.

\subsubsection{Radiation}

In the formulation of our framework we have neglected effects of radiation, which may tend to destabilize a cloud layer through longwave cooling at the top, and longwave heating at the bottom. We have excluded these effects because of the large range of possibilities resulting from variable solar insolation, and, particularly, underlying cloud cover. Further, the impact of radiation depends on the cloud's optical depth, which in turn depends on its entire vertical structure, and not just the atmospheric layer with depth $h$. Our results have shown that for low temperatures, cirrus lifetimes of at least several hours are to be expected. These lifetimes are long enough to allow radiation to affect the evolution of the cirrus cloud. While we do not expect that radiative effects would entirely change the results of, for example, dehydration efficiency, we stress that these limitations should be borne in mind when applying the results to the real atmosphere.

\subsubsection{Turbulence, wind shear and mixing}

The implicit assumption of a 0-dimensional model as employed here is that the box's integrity is not affected over the time of the model evaluation. Turbulent mixing and wind shear, however, might be expected to invalidate this assumption particularly for the cases with longer time scales $\tau_{T}$. While it is in principle possible to include effects of turbulent mixing with ambient air masses, one would have to introduce scenarios of the composition and temperature structure of these ambient air masses and the turbulent mixing time scale (which in turn might depend on the radiative heating/cooling of the cloud). Estimates of this time scale, based on measured turbulence spectra in the upper troposphere, vary over three orders of magnitude, ranging from minutes to many hours. Thus each case would have to be carefully assessed to determine the impact of turbulence on atmospheric dehydration via cirrus clouds. Such assessment is clearly beyond the scope of the treatment presented here, in which we present a limiting (zero-turbulence) case, yielding maximum water removal from the layer.

\section{Conclusions}

We have used a simple model to describe dehydration of an upper atmospheric layer by cirrus clouds that formed insitu. Scaling arguments show that the results can be applied to a broad range of parameter values. Expected variability/uncertainty of these parameters under atmospheric conditions, and model limitations as discussed in the text prevent applying the model results directly to specific observations. The model results do, however, provide general and useful insight into the dehydration potential of cirrus clouds in the upper troposphere.

The parameter combination $P$ that governs the impact of cirrus clouds on the air mass moisture budget, is essentially the ratio of the temperature perturbation time scale to that of the ice particle sedimentation. We find that under typical upper tropospheric conditions, dehydration is fast for $\tau_{\mathrm{T}}$ of order hours (and longer), or slow for shorter $\tau_{\mathrm{T}}$. More specifically, the results of our scaling study indicate that

a) for the range of the governing parameters under current conditions, the upper troposphere cannot hold substantially more water than given by the saturation mixing ratio based on the larger-scale temperature field (This result would change if $n_{0}$ were a factor 10 or more larger than under present conditions);

b) high frequency temperature perturbations $\tau_{\mathrm{T}}<\tau_{\text {crit. }}$ are too short to allow significant dehydration, with $\tau_{\text {crit. }}$ being temperature dependent. At $T_{\text {ref }}=235 \mathrm{~K} \tau_{\text {crit }} \approx 1 \mathrm{~h}$ and at $T_{\text {ref }}=205 \mathrm{~K} \tau_{\text {crit }} \approx 7 \mathrm{~h}$;

c) the dehydration efficiency decreases with increasing magnitude $\Delta T$ of the temperature perturbation for slow cases; it is independent of $\Delta T$ for fast cases.

The overall character of our results is independent of details of the ice nucleation mechanism, the cloud microphysics, the temperature fluctuation spectrum and/or the cloud depth. The major impact on our results of shifting any of these (for example, to higher nucleated ice particle density, to deeper clouds, or to a more complex representation of the particle size spectrum) is to simply shift the occurrence frequency of fast and slow regimes in the upper troposphere. For example, as $n_{0}$ increases strongly, the boundary between regimes is shifted in the $\tau_{\mathrm{T}} / T_{\text {ref }}$ space, as shown in Fig. 3, but the general character of $P$ and $\epsilon$ contours remains unchanged. Thus it may be argued that in a different climate regime, for example, in which $n_{0}$ and/or typical values of $\tau_{T}$ might change, the behaviors we have described would occur but in different regions of the atmosphere. In particular, the relationship between dehydration efficiency and $P$ given by 
Eq. (8) would hold in another climate regime; however, the values of $P$ and therefore of dehydration efficiency $\epsilon$ corresponding to fixed temperature and $\tau_{\mathrm{T}}$ would change.

Our model, in conjunction with data on the frequency distribution of clear air humidity, and spectra of temperature perturbations, could be used to estimate the distribution of $\tau_{\mathrm{T}}$ characteristic of the current upper troposphere. This would allow an assessment of the relative importance of the fast and slow regimes in today's atmosphere, a useful way to characterize the current state and predicted shifts in atmospheric behavior under changes in aerosols, greenhouse gases or other perturbations.

\section{Appendix A}

\section{The formalism}

\section{A1 Equations for cirrus model}

Let $Q(t)\left(q_{\text {ice }}(\mathrm{t})\right)$ be the total water (ice) mixing ratios $(\mathrm{kg}$ $\mathrm{H}_{2} \mathrm{O} / \mathrm{kg}$ air) in the cloud at time $t$ :

$Q(t)=q_{\mathrm{vapor}}(t)+q_{\text {ice }}(t)$

and let $n\left[\mathrm{~m}^{-3}\right](t)$ be the number density of ice crystals, all of the same radius $r$, in the cloud at time t.

Assume the temperature history:

$T(t) \equiv T_{\text {ref }}-\Delta T \cdot \sin \left(2 \pi \cdot t / \tau_{T}\right)$

where $\tau_{\mathrm{T}}$ is the period of the temperature oscillation, assumed isobaric, and $\Delta T[K]$ is its amplitude.

The cloud evolution equations are

$\frac{\mathrm{d} Q}{\mathrm{~d} t}=-q_{\text {ice }} \frac{v(r)}{h}, q_{\text {ice }}>0$,

and

$\frac{\mathrm{d} n}{\mathrm{~d} t}=-n \frac{v(r)}{h}$

where the sedimentation velocity of an ice particle of radius $r$ is: (Locatelli and Hobbs, 1974)

$v(r)=C \cdot r^{x}$.

$C$ and $x$ are constants depending on the flow regime (and consequently on the particle size), and $r$ is a measure of the particle size.

Now, we assume a monodisperse particle size distribution in the cloud layer (for discussion see Sect. 2), such that the ice water mixing ratio is approximately given by

$q_{\text {ice }}=n \frac{4 \pi}{3} \cdot \frac{\rho_{\text {ice }}}{\rho_{\text {air }}} \cdot r^{3} \equiv B(n) \cdot r^{3}$

where $\rho_{\text {ice }}$ is the density of solid ice and $\rho_{\text {air }}$ that of air at the given temperature and pressure. Expressing the particle radius and fall velocity in terms of condensed water content, particle number density and ratio of densities yields

$r=\left(q_{\text {ice }} / B(n)\right)^{1 / 3}, v(r)=C\left(q_{\text {ice }} / B(n)\right)^{x / 3}$

which we can insert into the time dependent equations for $Q$ and $n$ (Eqs. A3 and A4):

$$
\begin{aligned}
& \frac{\mathrm{d} Q}{\mathrm{~d} t}=-C \frac{q_{\mathrm{ice}}^{(1+x / 3)}}{h} \cdot\left(\frac{1}{B(n)}\right)^{x / 3} \\
& \frac{\mathrm{d} n}{\mathrm{~d} t}=-C \frac{n}{h} \cdot\left(\frac{q_{\text {ice }}}{B(n)}\right)^{x / 3} .
\end{aligned}
$$

Under the approximations of our model, we can express $Q$ and $q_{\text {ice }}$ as functions of the saturation point temperature $T^{*}$, defined for a layer of total water mixing ratio $Q$ at pressure $p$ as the temperature for which

$Q=q_{\mathrm{sat}}\left(T^{*}, p\right)$

where $q_{\mathrm{sat}}$ is the saturation mixing ratio over ice. For small perturbations in temperature $\delta T \equiv T-T_{\text {ref }}<<T_{\text {ref }}$ the Clausius-Clapeyron equation can be written

$q_{\mathrm{sat}}(T, p) \approx q_{\mathrm{sat}}\left(T_{\mathrm{ref}}, p\right) \exp \left(A\left(T_{\mathrm{ref}}\right) \cdot \delta T\right)$

where $A\left(T_{\text {ref }}\right) \equiv \frac{L_{\text {sub }}}{R_{v} T_{\text {ref }}^{2}}$ for $L_{\text {sub }}[\mathrm{J} / \mathrm{kg}], R_{v}[\mathrm{~J} / \mathrm{kg} / \mathrm{K}]$, being the latent heat of sublimation and the gas constant for vapor, respectively. $A\left(T_{\text {ref }}\right)$ varies betwee 0.1 and 0.14 over the temperature range considered here.

Then the water conservation equation (Eq. A3) becomes an equation for $\delta T^{*}$ :

$$
\begin{aligned}
\frac{\mathrm{d} \delta T^{*}}{\mathrm{~d} t} & =\frac{1}{A\left(T_{\mathrm{ref}}\right)} \frac{\mathrm{d} l n Q}{\mathrm{~d} t} \\
& =-\frac{1}{A\left(T_{\mathrm{ref}}\right) Q} \cdot C \frac{q_{\mathrm{ice}}^{(1+x / 3)}}{h} \cdot\left(\frac{1}{B(n)}\right)^{x / 3}
\end{aligned}
$$

and, assuming that in the presence of ice the cloudy air is exactly saturated with respect to ice,

$$
\begin{aligned}
q_{\text {ice }}(t) & =q_{\text {sat }}\left(T_{\text {ref }}+\delta T^{*}(t), p\right)-q_{\text {sat }}(T(t), p) \\
& \approx q_{\text {ice }, 0} \cdot(\delta \hat{T} *-\delta \hat{T})
\end{aligned}
$$

where we define

$q_{\text {ice }, 0} \equiv A\left(T_{\text {ref }}\right) \cdot \Delta T \cdot q_{\text {sat }}\left(T_{\text {ref }}\right)$.

It is convenient to nondimensionalize these equations. Let

$$
\begin{aligned}
\delta \hat{T}^{*} & \equiv \delta T^{*} / \Delta T \\
\delta \hat{T} & \equiv \delta T / \Delta T \\
\hat{t} & \equiv \frac{2 \pi t}{\tau_{T}} \\
\hat{n} & \equiv \frac{n}{n_{0}} \\
q_{\text {ice }} & \equiv \frac{q_{\text {ice }}}{q_{\text {ice }, 0}} \\
\hat{r} & \equiv \frac{r}{r_{0}}
\end{aligned}
$$


where the particle radius scale is

$r_{0} \equiv\left(\frac{q_{\text {ice }, 0}}{B\left(n_{0}\right)}\right)^{1 / 3}$.

The time scale for fall-out is

$\tau_{\text {fall }} \equiv \frac{h}{v\left(r_{0}\right)}$.

Note that $\tau_{\text {fall }}$ depends on $\Delta T$ and on $n_{0}$.

We define

$P \equiv \frac{\tau_{T}}{2 \pi \cdot \tau_{\text {fall }}}$.

The non-dimensionalized equations then are, from Eqs. (A8) and (A4),

$\frac{\mathrm{d} \hat{n}}{\mathrm{~d} \hat{t}}=-P \hat{q}_{\mathrm{ice}}^{x / 3} \hat{n}^{(1-x / 3)}$

and

$$
\begin{array}{r}
\frac{\mathrm{d}\left(\delta \hat{T}^{*}\right)}{\mathrm{d} \hat{t}}=- \\
-P \cdot \hat{q}_{\mathrm{ice}}^{(1+x / 3)} \cdot \hat{n}^{-x / 3} \times \\
\exp \left(-A\left(T_{\mathrm{ref}}\right) \Delta T \delta \hat{T}^{*}\right)
\end{array}
$$

Equations (A20) and (A21) constitute our model. The initial conditions are $\hat{n}(0)=1 ; \delta \hat{T}^{*}(0)=\delta T_{\text {nuc }}^{*} / \Delta T$, where $\delta T_{\text {nuc }}^{*}$ is that saturation temperature displacement required for nucleation.

The model equations and the initial conditions involve three parameters $T_{\text {ref }}, \Delta T$ and $P$. Over the range of temperatures considered here, $0.1 \leq A\left(T_{\text {ref }}\right) \leq 0.15$. Moreover, the nucleation temperature displacement remains in the range $3-3.2 \mathrm{~K}$ for $205 \mathrm{~K} \leq T_{\text {ref }} \leq 235 \mathrm{~K}$. Therefore the influence of variations in $T_{\text {ref }}$ on our results is very small. On the other hand, the temperature perturbation amplitude $\Delta T$ determines the initial condition, it comes into the equations (see Eq. A21) and it determines the velocity scale $v\left(r_{0}\right)$, so, indirectly, the third parameter, $P$. In most of the results presented in this paper we focus on the influence of $P$ at fixed $\Delta T$, and we deal with the impact of variations in $\Delta T$ in Sect. 4.1.

Acknowledgements. This publication is partially funded by the Joint Institute for the Study of the Atmosphere and Ocean (JISAO) under NOAA Cooperative Agreement No. NA17RJ1232, Contribution \# 1168. M. B. Baker is grateful for support by NSF ATM-021147. S. Fueglistaler is grateful for additional support by NASA grant NNG04GM23G.

Edited by: U. Lohmann

\section{References}

Betts, A. K.: Saturation Point Analysis of Moist Convective Overturning, J. Atmos. Sci., 39, 1484-1505, 1982.

Dessler, A. and Sherwood, S. C.: Simulations of tropical upper tropospheric humidity, J. Geophys. Res., 105, 20 155-20 163, 2000.

Dowling, D. R. and Radke, L. F.: A summary of the physical properties of cirrus clouds, J. Appl. Meteorol., 29(9), 970-978, 1990.

Fueglistaler, S., Luo, B. P., Voigt, C., Carslaw, K. S., and Peter, Th.: NAT-rock formation by mother clouds: a microphysical model study, Atmos. Phys. Chem., 2, 93-98, 2002.

Fueglistaler, S., Bonazzola, M., Haynes, P. H., and Peter, T.: Stratospheric water vapor predicted from the Lagrangian temperature history of air entering the stratosphere in the tropics, J. Geophys. Res., doi:10.1029/2004JD005516, 2005.

Hall, W. D. and Pruppacher, H. R.: The Survival of Ice Particles Falling from Cirrus Clouds in Subsaturated Air, J. Atmos. Sci., 33, 1995-2006, 1976.

Held, I. M. and Soden, B. J.: Water vapor feedback and global warming, Ann. Rev. En. Environ., 25, 411-475, 2000.

Hoyle, C., Luo, B. P., and Peter, T.: The Origin of High Ice Crystal Number Densities in Cirrus Clouds, J. Atmos. Sci., 62, 25682579, 2005.

Jensen, E. J., Toon, O. B., Selkirk, H. B., Spinhirne, J. D., and Schoeberl, M. R.: On the formation and persistence of subvisible cirrus clouds near the tropical tropopause, J. Geophys. Res., 101, $21361-21375,1996$.

Jensen, E. J. and Pfister, L.: Transport and Freeze-drying in the tropical tropopause layer, J. Geophys. Res., 109, D02207, doi:10.1029/2003JD004022, 2004.

Jensen, E. J., Pfister, L., Bui, T., Weinheimer, A., Weinstock, E., Smith, J., Pittman, J., Baumgardner, D., Lawson, P., and McGill, M. J.: Formation of a tropopause cirrus layer observed over Florida during CRYSTAL-FACE, J. Geophys. Res., 110, D03208, doi:10.1029/2004JD004671, 2005.

Kärcher, B. and Lohmann, U.: A parametrization of cirrus cloud formation: Homogeneous freezing of supercooled aerosols, J. Geophys. Res., 107, 4010, doi:10.1029/2001JD000470, 2002.

Koop, T., Luo, B. P., Tsias, A., and Peter, T.: Water activity as the determinant for homogeneous ice nucleation in aqueous solutions, Nature, 406, 611-614, 2000.

Locatelli, J. D. and Hobbs, P. V.: Fall speeds and masses of solid precipitation particles, J. Geophys. Res., 79, 2185-2197, 1974.

Luo, B. P., Peter, Th., Wernli, H., et al.: Ultrathin Tropical Tropopause Clouds (UTTCs): II. Stabilization mechanisms, Atmos. Chem. Phys., 3, 1093-1100, 2003.

Pierrehumbert, R. T.: Lateral mixing as a source of subtropical water vapor, Geophys. Res. Lett., 25, 151-154, 1998. 